\title{
On the harmonic and hyperharmonic Fibonacci numbers
}

Naim Tuglu' ${ }^{*}$, Can Kızılateş ${ }^{2}$ and Seyhun Kesim²

"Correspondence:

naimtuglu@gazi.edu.tr

'Department of Mathematics, Gazi University, Teknikokullar, Ankara,

06500, Turkey

Full list of author information is

available at the end of the article

\begin{abstract}
In this paper, we study the theory of the harmonic and the hyperharmonic Fibonacci numbers. Also, we get some combinatoric identities like as harmonic and hyperharmonic numbers and we obtain some useful formulas for $\mathbb{F}_{n}$, which is concerned with finite sums of reciprocals of Fibonacci numbers. We obtain the spectral and Euclidean norms of circulant matrices involving harmonic and hyperharmonic Fibonacci numbers.
\end{abstract}

MSC: Primary 11B39; 05A19; secondary 05A10; 15A60

Keywords: harmonic number; hyperharmonic number; harmonic Fibonacci number; hyperharmonic Fibonacci number; matrix norm

\section{Introduction}

The harmonic numbers have many applications in combinatorics and other areas. Many authors have studied these numbers. The $n$th harmonic number, $H_{n}$, is defined by

$$
H_{n}=\sum_{k=1}^{n} \frac{1}{k}
$$

where $H_{0}=0$. The $n$th harmonic number $H_{n}$ can be expressed as

$$
H_{n}=\frac{\left[\begin{array}{c}
n+1 \\
2
\end{array}\right]}{n !},
$$

where $\left[\begin{array}{l}n \\ k\end{array}\right]$ denotes the Stirling number of the first kind, counting the permutations of $n$ elements that are the product of $k$ disjoint cycles.

Several interesting properties of harmonic numbers can be found in [1]. For $n \geq 1$, some of them are the following:

$$
\begin{aligned}
& \sum_{k=1}^{n-1} H_{k}=n H_{n}-n, \\
& \sum_{k=1}^{n-1}\left(\begin{array}{c}
k \\
m
\end{array}\right) H_{k}=\left(\begin{array}{c}
n \\
m+1
\end{array}\right)\left(H_{n}-\frac{1}{m+1}\right), \\
& \sum_{k=1}^{n-1} k H_{k}=\frac{n^{2}}{2}\left(H_{n}-\frac{1}{2}\right),
\end{aligned}
$$

(c) 2015 Tuglu et al. This article is distributed under the terms of the Creative Commons Attribution 4.0 International License (http://creativecommons.org/licenses/by/4.0/), which permits unrestricted use, distribution, and reproduction in any medium, provided you give appropriate credit to the original author(s) and the source, provide a link to the Creative Commons license, and indicate if changes were made. 
where $n^{\underline{2}}=n(n-1)$ and $m$ is a nonnegative integer.

In [2], Spivey proved that, for $n \geq 1$,

$$
\begin{aligned}
& \sum_{k=0}^{n}\left(\begin{array}{l}
n \\
k
\end{array}\right) H_{k}=2^{n}\left(H_{n}-\sum_{k=1}^{n} \frac{1}{k 2^{k}}\right), \\
& \sum_{k=0}^{n}\left(\begin{array}{l}
n \\
k
\end{array}\right)(-1)^{k} H_{k}=-\frac{1}{n} .
\end{aligned}
$$

Harmonic numbers have been generalized by many authors [3-7]. In [5], Conway and Guy defined the $n$th hyperharmonic number of order $r, H_{n}^{(r)}$ for $n, r \geq 1$ by the following recurrence relations:

$$
H_{n}^{(r)}=\sum_{k=1}^{n} H_{k}^{(r-1)},
$$

where $H_{n}^{(0)}=\frac{1}{n}$ and $H_{n}^{(1)}=H_{n}$ is the $n$th ordinary harmonic number.

Furthermore, these numbers can be expressed by binomial coefficients and ordinary harmonic numbers [5], as follows:

$$
H_{n}^{(r)}=\left(\begin{array}{c}
n+r-1 \\
r-1
\end{array}\right)\left(H_{n+r-1}-H_{r-1}\right)
$$

In [4], Benjamin et al. gave the following properties of hyperharmonic numbers:

$$
\begin{aligned}
& H_{n}^{(r)}=\sum_{t=1}^{n}\left(\begin{array}{c}
n+r-t-1 \\
r-1
\end{array}\right) \frac{1}{t}, \\
& H_{n}^{(r)}=\sum_{t=1}^{n}\left(\begin{array}{c}
n+r-m-t-1 \\
r-m-1
\end{array}\right) H_{t}^{(m)},
\end{aligned}
$$

where $0 \leq m \leq r-1$. In [3], Bahsi and Solak defined a special matrix whose entries are hyperharmonic numbers and gave some properties of this matrix.

The Fibonacci sequence is defined by the following recurrence relation, for $n \geq 0$ :

$$
F_{n+2}=F_{n+1}+F_{n}
$$

with $F_{0}=0, F_{1}=1$.

In [8], Dil and Mezö defined hyper-Fibonacci numbers, $F_{n}^{(r)}$, for positive integer $r$,

$$
F_{n}^{(r)}=\sum_{k=0}^{n} F_{k}^{(r-1)}
$$

with $F_{n}^{(0)}=F_{n}, F_{0}^{(r)}=0$, and $F_{1}^{(r)}=1$. Moreover, the authors obtained some properties of these numbers.

In [9], Ohtsuka and Nakamura studied the partial infinite sums of reciprocals Fibonacci numbers and the reciprocal of the square of the Fibonacci numbers. Holiday and Komatsu generalized their results in [10]. 
The important point to note here is the form of finite sums of reciprocals Fibonacci numbers. In [11], Rabinowitz pointed out that

$$
\mathbb{F}_{n}=\sum_{k=1}^{n} \frac{1}{F_{k}}
$$

is no known simple form. Now we present some preliminaries to our study.

In [1], the difference operator of $f(x)$ is defined as

$$
\Delta f(x)=f(x+1)-f(x)
$$

The expression $x$ to the $m$ falling is denoted $x^{\underline{\underline{m}}}$. The value of

$$
x^{\underline{m}}=x(x-1)(x-2) \cdots(x-m+1)
$$

and it is called the falling power.

The $\Delta$ operator has a very interesting property for $m \geq 0$,

$$
\Delta x^{\underline{m}}=m x^{\underline{m-1}} .
$$

Analogously, the $\Delta$ operator has an inverse, the anti-difference or summation operator $\sum$ defined as follows. If $\Delta f(x)=g(x)$ then

$$
\sum_{a}^{b} g(x) \delta_{x}=\sum_{x=a}^{b-1} g(x)=f(b)-f(a) .
$$

The anti-difference operator $\sum$ has some properties as follows:

$$
\sum x^{\underline{m}} \delta_{x}= \begin{cases}\frac{x^{\underline{m+1}}}{m+1} & \text { if } m \neq-1 \\ H_{x} & \text { if } m=-1\end{cases}
$$

and

$$
\sum_{a}^{b} u(x) \Delta v(x) \delta_{x}=\left.u(x) v(x)\right|_{a} ^{b+1}-\sum_{a}^{b} E v(x) \Delta u(x) \delta_{x},
$$

where $E v(x)=v(x+1)[1]$.

In [1] the authors use a property of the finite difference operator to show the validity of the identity $(1)$ as follows.

Let $u(k)=H_{k}$ and $\Delta v(k)=1$ be as in (6). Then they obtain $\Delta u(k)=\frac{1}{k+1}$ and $v(k)=k$. Therefore,

$$
\begin{aligned}
\sum_{k=1}^{n-1} H_{k} & =\left.k H_{k}\right|_{1} ^{n}-\sum_{1}^{n}(k+1) \frac{1}{k+1} \delta_{k} \\
& =n H_{n}-n .
\end{aligned}
$$

In this paper, inspired by the definition of a harmonic number, we introduce harmonic Fibonacci numbers and give various identities for these numbers by using the difference 
operator. Moreover, we introduce hyperharmonic Fibonacci numbers and present some certain properties of them; the same as hyperharmonic numbers. Finally, we obtain the spectral and the Euclidean norms of circulant matrices with harmonic and hyperharmonic Fibonacci numbers.

\section{Harmonic Fibonacci numbers and some combinatorial properties}

In this section, we investigate various properties of $\mathbb{F}_{n}$; finite sums of the reciprocals of the Fibonacci numbers are defined as

$$
\mathbb{F}_{n}=\sum_{k=1}^{n} \frac{1}{F_{k}}
$$

Here and subsequently we call it the $n$th harmonic Fibonacci number. Now we state some theorems related to harmonic Fibonacci numbers.

Theorem 1 Let $\mathbb{F}_{n}$ be the nth harmonic Fibonacci number. Then we have

$$
\sum_{k=0}^{n-1} \mathbb{F}_{k}=n \mathbb{F}_{n}-\sum_{k=0}^{n-1} \frac{k+1}{F_{k+1}} .
$$

Proof Our proof starts with the observation of a property of the difference operator. Let $u(k)=\mathbb{F}_{k}$ and $\Delta v(k)=1$ be as in (6). Then we obtain $\Delta u(k)=\frac{1}{F_{k+1}}, v(k)=k$ and $E v(k)=k+1$. Hence, we have

$$
\sum_{k=0}^{n-1} \mathbb{F}_{k}=n \mathbb{F}_{n}-\sum_{k=0}^{n-1} \frac{k+1}{F_{k+1}} .
$$

Theorem 2 Let $\mathbb{F}_{n}$ be the nth harmonic Fibonacci number. Then we have

$$
\sum_{k=0}^{n-1}\left(\mathbb{F}_{k}\right)^{2}=n \mathbb{F}_{n}^{2}-\sum_{k=0}^{n-1} \frac{k+1}{F_{k+1}}\left(2 \mathbb{F}_{k}+\frac{1}{F_{k+1}}\right) .
$$

Proof Let $u(k)=\mathbb{F}_{k}^{2}$ and $\Delta v(k)=1$ be as in (6). Then by using (6), we obtain

$$
\sum_{k=0}^{n-1}\left(\mathbb{F}_{k}\right)^{2}=n \mathbb{F}_{n}^{2}-\sum_{k=0}^{n-1} \frac{k+1}{F_{k+1}}\left(2 \mathbb{F}_{k}+\frac{1}{F_{k+1}}\right) .
$$

Theorem 3 Let $\mathbb{F}_{n}$ be the nth harmonic Fibonacci number and $m$ be a nonnegative integer, we have

$$
\sum_{k=0}^{n-1}\left(\begin{array}{c}
k \\
m
\end{array}\right) \mathbb{F}_{k}=\left(\begin{array}{c}
n \\
m+1
\end{array}\right) \mathbb{F}_{n}-\sum_{k=0}^{n-1}\left(\begin{array}{c}
k+1 \\
m+1
\end{array}\right) \frac{1}{F_{k+1}}
$$

Proof Let $u(k)=\mathbb{F}_{k}$ and $\Delta v(k)=\left(\begin{array}{l}k \\ m\end{array}\right)$ be as in (6). Then we obtain $\Delta u(k)=\frac{1}{F_{k+1}}, v(k)=\left(\begin{array}{c}k \\ m+1\end{array}\right)$, and $E v(k)=\left(\begin{array}{c}k+1 \\ m+1\end{array}\right)$. By using (6), we have

$$
\sum_{k=0}^{n-1}\left(\begin{array}{c}
k \\
m
\end{array}\right) \mathbb{F}_{k}=\left(\begin{array}{c}
n \\
m+1
\end{array}\right) \mathbb{F}_{n}-\sum_{k=0}^{n-1}\left(\begin{array}{c}
k+1 \\
m+1
\end{array}\right) \frac{1}{F_{k+1}}
$$


Theorem 4 Let $\mathbb{F}_{n}$ be the nth harmonic Fibonacci number and $m$ be a nonnegative integer. Then we have

$$
\sum_{k=1}^{n-1} k^{\frac{m}{m}} \mathbb{F}_{k}=\frac{n \frac{m+1}{m+1}}{m} \mathbb{F}_{n}-\sum_{k=0}^{n-1} \frac{(k+1)^{\frac{m+1}{}}}{m+1} \frac{1}{F_{k+1}} .
$$

Proof Let $u(k)=\mathbb{F}_{k}$ and $\Delta v(k)=k^{\underline{m}}$ be as in (6). Then we get $\Delta u(k)=\frac{1}{F_{k+1}}, v(k)=\frac{k^{\underline{m+1}}}{m+1}$, and $E v(k)=\frac{(k+1) \underline{m+1}}{m+1}$. With the aid of (6), we have

$$
\sum_{k=1}^{n-1} k^{\underline{m}} \mathbb{F}_{k}=\frac{n^{\frac{m+1}{m}}}{m+1} \mathbb{F}_{n}-\sum_{k=0}^{n-1} \frac{(k+1)^{\frac{m+1}{2}}}{m+1} \frac{1}{F_{k+1}} .
$$

The following theorem gives the relationship between harmonic numbers and harmonic Fibonacci numbers.

Theorem 5 Let $\mathbb{F}_{n}$ be the nth harmonic Fibonacci number, we have

$$
\sum_{k=0}^{n-1} \frac{\mathbb{F}_{k}}{k+1}=H_{n} \mathbb{F}_{n}-\sum_{k=0}^{n-1} \frac{H_{k+1}}{F_{k+1}} .
$$

Proof Let $u(k)=\mathbb{F}_{k}$ and $\Delta v(k)=\frac{1}{k+1}$ be as in (6). Then we obtain $\Delta u(k)=\frac{1}{F_{k+1}}, v(k)=H_{k}$, and $E v(k)=H_{k+1}$. By using (6), we have

$$
\sum_{k=0}^{n-1} \frac{\mathbb{F}_{k}}{k+1}=H_{n} \mathbb{F}_{n}-\sum_{k=0}^{n-1} \frac{H_{k+1}}{F_{k+1}} .
$$

\section{Corollary 1}

$$
\sum_{k=0}^{n-1} \frac{\mathbb{F}_{k}}{k+1}=\frac{\left[\begin{array}{c}
n+1 \\
2
\end{array}\right]}{n !} \mathbb{F}_{n}-\sum_{k=0}^{n-1} \frac{\left[\begin{array}{c}
k+2 \\
2
\end{array}\right]}{(k+1) ! F_{k+1}}
$$

Proof If we take $H_{n}=\frac{\left[\begin{array}{c}n+1 \\ n !\end{array}\right]}{n !}$ in Theorem 5 the proof can be completed.

Theorem 6 We have

$$
\sum_{k=0}^{n-1} F_{k-1} \mathbb{F}_{k}=F_{n} \mathbb{F}_{n}-n
$$

Proof Let $u(k)=\mathbb{F}_{k}$ and $\Delta v(k)=F_{k-1}$ be as in (6). Then we obtain $\Delta u(k)=\frac{1}{F_{k+1}}, v(k)=F_{k}$, and $E v(k)=F_{k+1}$. By using (6), we have

$$
\begin{aligned}
\sum_{k=0}^{n-1} F_{k-1} \mathbb{F}_{k} & =F_{n} \mathbb{F}_{n}-\sum_{k=0}^{n-1} k^{0} \delta_{k} \\
& =F_{n} \mathbb{F}_{n}-n .
\end{aligned}
$$




\section{Hyperharmonic Fibonacci numbers}

In this section, hyperharmonic Fibonacci numbers will be defined having used a similarity and affiliation between harmonic numbers and hyperharmonic numbers with the help of harmonic Fibonacci numbers. Now we define $\mathbb{F}_{n}^{(r)}$, the hyperharmonic Fibonacci numbers of order $r$.

Definition 1 Let $\mathbb{F}_{n}$ be the $n$th harmonic Fibonacci number. For $n, r \geq 1$ hyperharmonic Fibonacci numbers are defined by

$$
\mathbb{F}_{n}^{(r)}=\sum_{k=1}^{n} \mathbb{F}_{k}^{(r-1)}
$$

with $\mathbb{F}_{n}^{(0)}=\frac{1}{F_{n}}$ and $\mathbb{F}_{0}^{(k)}=0$ for $k \geq 0$.

In particular for $r=1$ we get

$$
\mathbb{F}_{n}^{(1)}=\mathbb{F}_{n}=\sum_{k=1}^{n} \frac{1}{F_{k}},
$$

where $\mathbb{F}_{n}$ is $n$th harmonic Fibonacci numbers.

Lemma 1 Hyperharmonic Fibonacci numbers have a recurrence relation as follows:

$$
\mathbb{F}_{n}^{(r)}=\mathbb{F}_{n}^{(r-1)}+\mathbb{F}_{n-1}^{(r)}
$$

Proof From (8) we have

$$
\begin{aligned}
\mathbb{F}_{n}^{(r)} & =\sum_{k=1}^{n} \mathbb{F}_{k}^{(r-1)} \\
& =\sum_{k=1}^{n-1} \mathbb{F}_{k}^{(r-1)}+\mathbb{F}_{n}^{(r-1)} \\
& =\mathbb{F}_{n-1}^{(r)}+\mathbb{F}_{n}^{(r-1)} .
\end{aligned}
$$

We give an interesting property of hyperharmonic numbers; the same as in (4).

Theorem 7 For $1 \leq i, j \leq n$, we have

$$
\mathbb{F}_{n-i+1}^{(j)}=\sum_{k=i}^{n}\left(\begin{array}{c}
n-k+j-1 \\
j-1
\end{array}\right) \frac{1}{F_{k-i+1}} .
$$

Proof We begin by recalling the definition of $\mathbb{F}_{n}^{(r)}$. If we use this definition $j-1$ times, we get

$$
\begin{aligned}
\mathbb{F}_{n-i+1}^{(j)} & =\sum_{k=1}^{n-i+1} \mathbb{F}_{k}^{(j-1)} \\
& =\sum_{k_{j}=1}^{n-i+1} \sum_{k_{j-1}=1}^{k_{j}} \cdots \sum_{k_{1}=1}^{k_{2}} \frac{1}{F_{k_{1}}} .
\end{aligned}
$$


We use induction on $n$ to obtain

$$
\sum_{k_{j}=1}^{n-i+1} \sum_{k_{j-1}=1}^{k_{j}} \cdots \sum_{k_{1}=1}^{k_{2}} \frac{1}{F_{k_{1}}}=\sum_{k=i}^{n}\left(\begin{array}{c}
n-k+j-1 \\
j-1
\end{array}\right) \frac{1}{F_{k-i+1}} .
$$

Clearly it is true for $n=1$. Suppose it is true for some $n>1$, then using the induction hypothesis, we have

$$
\begin{aligned}
& \sum_{k_{j}=1}^{n-i+2} \sum_{k_{j-1}=1}^{k_{j}} \cdots \sum_{k_{1}=1}^{k_{2}} \frac{1}{F_{k_{1}}}=\sum_{k_{j}=1}^{n-i+1} \sum_{k_{j-1}=1}^{k_{j}} \cdots \sum_{k_{1}=1}^{k_{2}} \frac{1}{F_{k_{1}}}+\sum_{k_{j-1}=1}^{n-i+2} \sum_{k_{j-2}=1}^{k_{j-1}} \cdots \sum_{k_{1}=1}^{k_{2}} \frac{1}{F_{k_{1}}} \\
& =\sum_{k_{j}=1}^{n-i+1} \sum_{k_{j-1}=1}^{k_{j}} \cdots \sum_{k_{1}=1}^{k_{2}} \frac{1}{F_{k_{1}}}+\sum_{k_{j-1}=1}^{n-i+1} \sum_{k_{j-2}=1}^{k_{j-1}} \cdots \sum_{k_{1}=1}^{k_{2}} \frac{1}{F_{k_{1}}} \\
& +\sum_{k_{j-2}=1}^{n-i+2} \sum_{k_{j-3}=1}^{k_{j-2}} \cdots \sum_{k_{1}=1}^{k_{2}} \frac{1}{F_{k_{1}}} \\
& =\sum_{k_{j}=1}^{n-i+1} \sum_{k_{j-1}=1}^{k_{j}} \cdots \sum_{k_{1}=1}^{k_{2}} \frac{1}{F_{k_{1}}}+\sum_{k_{j-1}=1}^{n-i+1} \sum_{k_{j-2}=1}^{k_{j-1}} \cdots \sum_{k_{1}=1}^{k_{2}} \frac{1}{F_{k_{1}}}+\cdots \\
& +\sum_{k_{2}=1}^{n-i+1} \sum_{k_{1}=1}^{k_{2}} \frac{1}{F_{k_{1}}}+\sum_{k_{1}=1}^{n-i+2} \frac{1}{F_{k_{1}}} \\
& =\sum_{k_{j}=1}^{n-i+1} \sum_{k_{j-1}=1}^{k_{j}} \cdots \sum_{k_{1}=1}^{k_{2}} \frac{1}{F_{k_{1}}}+\sum_{k_{j-1}=1}^{n-i+1} \sum_{k_{j-2}=1}^{k_{j-1}} \cdots \sum_{k_{1}=1}^{k_{2}} \frac{1}{F_{k_{1}}}+\cdots \\
& +\sum_{k_{2}=1}^{n-i+1} \sum_{k_{1}=1}^{k_{2}} \frac{1}{F_{k_{1}}}+\sum_{k_{1}=1}^{n-i+1} \frac{1}{F_{k_{1}}}+\frac{1}{F_{n-i+2}} \\
& =\sum_{k=i}^{n} \frac{1}{F_{k-i+1}}\left[\left(\begin{array}{c}
n-k+j-1 \\
j-1
\end{array}\right)+\left(\begin{array}{c}
n-k+j-2 \\
j-2
\end{array}\right)+\cdots+\left(\begin{array}{c}
n-k \\
0
\end{array}\right)\right] \\
& +\frac{1}{F_{n-i+2}} \\
& =\sum_{k=i}^{n} \frac{1}{F_{k-i+1}}\left(\begin{array}{c}
n-k+j \\
j-1
\end{array}\right)+\frac{1}{F_{n-i+2}} \\
& =\sum_{k=i}^{n+1}\left(\begin{array}{c}
n-k+j \\
j-1
\end{array}\right) \frac{1}{F_{k-i+1}} \text {. }
\end{aligned}
$$

Finally we obtain

$$
\mathbb{F}_{n-i+1}^{(j)}=\sum_{k=i}^{n}\left(\begin{array}{c}
n-k+j-1 \\
j-1
\end{array}\right) \frac{1}{F_{k-i+1}} .
$$

\section{Corollary 2 We have}

$$
\mathbb{F}_{n}^{(r)}=\sum_{k=1}^{n}\left(\begin{array}{c}
n-k+r-1 \\
r-1
\end{array}\right) \frac{1}{F_{k}} .
$$


Proof By putting $i=1$ and $j=r$ in Theorem 7 , one has

$$
\mathbb{F}_{n}^{(r)}=\sum_{k=1}^{n}\left(\begin{array}{c}
n-k+r-1 \\
r-1
\end{array}\right) \frac{1}{F_{k}}
$$

At this point, we express $\mathbb{F}_{n}^{(r+s)}$ in terms of $\mathbb{F}_{1}^{(s)}, \mathbb{F}_{2}^{(s)}, \ldots, \mathbb{F}_{n}^{(s)}$ with the following theorem.

Theorem 8 For $r \geq 1$ and $s \geq 0$ we have

$$
\mathbb{F}_{n}^{(r+s)}=\sum_{t=1}^{n}\left(\begin{array}{c}
n-t+r-1 \\
r-1
\end{array}\right) \mathbb{F}_{t}^{(s)}
$$

Proof We prove this by induction on $n$. Clearly it is true for $n=1$. Assuming (10) to hold for $n>1$, we will prove it for $n+1$. Thus,

$$
\begin{aligned}
\mathbb{F}_{n+1}^{(r+s)}= & \mathbb{F}_{n+1}^{(r+s-1)}+\mathbb{F}_{n}^{(r+s)} \\
= & \mathbb{F}_{n+1}^{(r+s-2)}+\mathbb{F}_{n}^{(r+s-1)}+\mathbb{F}_{n}^{(r+s)} \\
& \vdots \\
= & \mathbb{F}_{n+1}^{(s)}+\mathbb{F}_{n}^{(s+1)}+\cdots+\mathbb{F}_{n}^{(r+s-1)}+\mathbb{F}_{n}^{(r+s)} \\
= & \mathbb{F}_{n+1}^{(s)}+\sum_{t=1}^{n}\left[\left(\begin{array}{c}
n-t \\
0
\end{array}\right)+\left(\begin{array}{c}
n-t+1 \\
1
\end{array}\right)+\cdots+\left(\begin{array}{c}
n-r-t-1 \\
r-1
\end{array}\right)\right] \mathbb{F}_{t}^{(s)} \\
= & \mathbb{F}_{n+1}^{(s)}+\sum_{t=1}^{n}\left(\begin{array}{c}
n-t+r \\
r-1
\end{array}\right) \mathbb{F}_{t}^{(s)} \\
& \sum_{t=1}^{n+1}\left(\begin{array}{c}
n-t+r \\
r-1
\end{array}\right) \mathbb{F}_{t}^{(s)} .
\end{aligned}
$$

Thus the proof is completed by the mathematical induction.

Another proof Let $C_{n}^{(r)}$ be the $n \times n$ matrix which is defined by

$$
C_{n}^{(r)}=\left(\begin{array}{cccc}
\mathbb{F}_{n}^{(r)} & \mathbb{F}_{n}^{(r+1)} & \cdots & \mathbb{F}_{n}^{(r+n-1)} \\
\mathbb{F}_{n-1}^{(r)} & \mathbb{F}_{n-1}^{(r+1)} & \cdots & \mathbb{F}_{n-1}^{(r+n-1)} \\
\vdots & \vdots & \cdots & \vdots \\
\mathbb{F}_{1}^{(r)} & \mathbb{F}_{1}^{(r+1)} & \cdots & \mathbb{F}_{1}^{(r+n-1)}
\end{array}\right)
$$

where $\mathbb{F}_{n}^{(r)}$ is the $n$th hyperharmonic Fibonacci number. Let

$$
A=\left(\begin{array}{cccc}
1 & 1 & \cdots & 1 \\
0 & 1 & \cdots & 1 \\
\vdots & \vdots & \ddots & \vdots \\
0 & 0 & \cdots & 1
\end{array}\right)
$$


be an $n \times n$ upper triangle matrix. In [3], Bahsi and Solak show that $A^{r}=\left(b_{i j}\right)_{n \times n}$, where

$$
b_{i j}= \begin{cases}\left(\begin{array}{c}
j+i+r-1 \\
r-1
\end{array}\right) & \text { if } i \leq j, \\
0 & \text { otherwise. }\end{cases}
$$

From the matrix multiplication one obtains

$$
C_{n}^{(r+s)}=A^{r} C_{n}^{(s)}
$$

Hence the element of $\left(C_{n}^{(r+s)}\right)_{11}$ is

$$
\begin{aligned}
\mathbb{F}_{n}^{(r+s)} & =\sum_{j=1}^{n} b_{1 j} \mathbb{F}_{n-j+1}^{(s)} \\
& =\sum_{j=1}^{n}\left(\begin{array}{c}
j+r-2 \\
r-1
\end{array}\right) \mathbb{F}_{n-j+1}^{(s)} \\
& =\sum_{t=1}^{n}\left(\begin{array}{c}
n-t+r-1 \\
r-1
\end{array}\right) \mathbb{F}_{t}^{(s)} .
\end{aligned}
$$

\section{An application of harmonic and hyperharmonic Fibonacci numbers in circulant matrices}

In this section, we will give some applications on matrix norms of harmonic Fibonacci and hyperharmonic Fibonacci numbers. Recently, there have been many papers on the norms of circulant matrices with special numbers [12-15].

Let $A=\left(a_{i j}\right)$ be any $m \times n$ complex matrix. The Euclidean norm and the spectral norm of the matrix $A$ are, respectively,

$$
\|A\|_{E}=\left(\sum_{i=1}^{m} \sum_{j=1}^{n}\left|a_{i j}\right|^{2}\right)^{\frac{1}{2}}
$$

and

$$
\|A\|_{2}=\sqrt{\max _{1 \leq i \leq n}\left|\lambda_{i}\left(A^{H} A\right)\right|},
$$

where $\lambda_{i}\left(A^{H} A\right)$ is an eigenvalue of $A^{H} A$ and $A^{H}$ is the conjugate transpose of the matrix $A$. Then the following inequality holds:

$$
\|A\|_{2} \leq\|A\|_{E} \leq \sqrt{n}\|A\|_{2}
$$

By a circulant matrix of order $n$ is meant a square matrix of the form

$$
C=\operatorname{Circ}\left(c_{0}, c_{1}, \ldots, c_{n-1}\right)=\left(\begin{array}{ccccc}
c_{0} & c_{1} & c_{2} & \cdots & c_{n-1} \\
c_{n-1} & c_{0} & c_{1} & \cdots & c_{n-2} \\
\vdots & \vdots & \vdots & & \vdots \\
c_{1} & c_{2} & c_{3} & \cdots & c_{0}
\end{array}\right)
$$


Theorem 9 Let $C_{1}=\operatorname{Circ}\left(\mathbb{F}_{0}, \mathbb{F}_{1}, \mathbb{F}_{2}, \ldots, \mathbb{F}_{n-1}\right)$ be an $n \times n$ circulant matrix. The Euclidean norm of $C_{1}$ is

$$
\left\|C_{1}\right\|_{E}=\left[n^{2} \mathbb{F}_{n}^{2}-n \sum_{k=0}^{n-1} \frac{k+1}{F_{k+1}}\left(2 \mathbb{F}_{k}+\frac{1}{F_{k+1}}\right)\right]^{\frac{1}{2}} .
$$

Proof From the definition of the Euclidean norm, we have

$$
\left\|C_{1}\right\|_{E}^{2}=n \sum_{k=0}^{n-1} \mathbb{F}_{k}^{2}
$$

Then by using (7), we have

$$
\left\|C_{1}\right\|_{E}=\left[n^{2} \mathbb{F}_{n}^{2}-n \sum_{k=0}^{n-1} \frac{k+1}{F_{k+1}}\left(2 \mathbb{F}_{k}+\frac{1}{F_{k+1}}\right)\right]^{\frac{1}{2}} .
$$

For the proof of the following theorem, we use the same method as in [12].

Theorem 10 Let $C_{1}=\operatorname{Circ}\left(\mathbb{F}_{0}, \mathbb{F}_{1}, \mathbb{F}_{2}, \ldots, \mathbb{F}_{n-1}\right)$ be an $n \times n$ circulant matrix. The spectral norm of $C_{1}$ is

$$
\left\|C_{1}\right\|_{2}=n \mathbb{F}_{n}-\sum_{k=0}^{n-1} \frac{k+1}{F_{k+1}} .
$$

Proof Since the circulant matrices are normal, the spectral norm of the circulant $C_{1}$ is equal to its spectral radius. Furthermore, $C_{1}$ is irreducible and its entries are nonnegative, we see that the spectral radius of the matrix $C_{1}$ is equal to its Perron root. Let $v$ be a vector with all components 1 . Then

$$
C_{1} v=\left(\sum_{k=0}^{n-1} \mathbb{F}_{k}\right) v
$$

Obviously, $\sum_{k=0}^{n-1} \mathbb{F}_{k}$ is an eigenvalue of $C_{1}$. Corresponding to a positive eigenvector, it must be the Perron root of the matrix $C_{1}$. Hence from Theorem 1, we have

$$
\left\|C_{1}\right\|_{2}=n \mathbb{F}_{n}-\sum_{k=0}^{n-1} \frac{k+1}{F_{k+1}} .
$$

Theorem 11 Let $C_{2}=\operatorname{Circ}\left(\mathbb{F}_{0}^{(r)}, \mathbb{F}_{1}^{(r)}, \mathbb{F}_{2}^{(r)}, \ldots, \mathbb{F}_{n-1}^{(r)}\right)$ be an $n \times n$ circulant matrix. The spectral norm of $C_{2}$ is

$$
\left\|C_{2}\right\|_{2}=\mathbb{F}_{n-1}^{(r+1)}
$$

Proof An analysis similar to that in the proof of Theorem 10 shows that

$$
\left\|C_{2}\right\|_{2}=\sum_{k=0}^{n-1} \mathbb{F}_{k}^{(r)} .
$$


From the definition of hyperharmonic Fibonacci numbers, we have

$$
\left\|C_{2}\right\|_{2}=\mathbb{F}_{n-1}^{(r+1)}
$$

Corollary 3 For the Euclidean norm of the matrix $C_{2}=\operatorname{Circ}\left(\mathbb{F}_{0}^{(r)}, \mathbb{F}_{1}^{(r)}, \mathbb{F}_{2}^{(r)}, \ldots, \mathbb{F}_{n-1}^{(r)}\right)$, we have

$$
\mathbb{F}_{n-1}^{(r+1)} \leq\left\|C_{2}\right\|_{E} \leq \sqrt{n} \mathbb{F}_{n-1}^{(r+1)}
$$

Proof The proof is trivial from Theorem 11 and the relation between the spectral norm and the Euclidean norm in (11).

Corollary 4 For the sum of the squares of hyperharmonic Fibonacci numbers, we have

$$
\frac{1}{\sqrt{n}} \mathbb{F}_{n-1}^{(r+1)} \leq \sqrt{\sum_{k=0}^{n-1}\left(\mathbb{F}_{k}^{(r)}\right)^{2}} \leq \mathbb{F}_{n-1}^{(r+1)} .
$$

Proof It is easily seen from the definition of the Euclidean norm and Corollary 3.

\section{Conclusion}

Our results can be applied to any linear recurrence sequences by using a similar method; for example, Lucas numbers, Pell numbers, Horadam numbers, generalized Fibonacci $p$-numbers.

\section{Competing interests}

The authors declare that they have no competing interests.

\section{Authors' contributions}

Each of the authors contributed to each part of this work equally and read and approved the final version of the manuscript.

\section{Author details}

'Department of Mathematics, Gazi University, Teknikokullar, Ankara, 06500, Turkey. ${ }^{2}$ Department of Mathematics, Bülent Ecevit University, Incivez, Zonguldak, 67100, Turkey.

\section{Acknowledgements}

The authors are grateful to anonymous referees and the associate editor for their careful reading, helpful comments and constructive suggestions, which improved the presentation of the results.

Received: 5 March 2015 Accepted: 4 September 2015 Published online: 17 September 2015

\section{References}

1. Graham, R, Knuth, D, Patashnik, K: Concrete Mathematics. Addison-Wesley, Reading (1989)

2. Spivey, MZ: Combinatorial sums and finite differences. Discrete Math. 307, 3130-3146 (1975)

3. Bahsi, M, Solak, S: An application of hyperharmonic numbers in matrices. Hacet. J. Math. Stat. 42, 387-393 (2013)

4. Benjamin, AT, Gaebler, D, Gaebler, R: A combinatorial approach to hyperharmonic numbers. Integers 3, A15 (2003)

5. Conway, JH, Guy, RK: The Book of Numbers. Springer, New York (1996)

6. Kronenburg, MJ: Some generalized harmonic number identities. arXiv:1103.5430

7. Cheon, GS, Mikkawy, MEA: Generalized harmonic number identities and a related matrix representation. J. Korean Math. Soc. 44, 487-498 (2007)

8. Dil, A, Mezö, I: A symmetric algorithm for hyperharmonic and Fibonacci numbers. Appl. Math. Comput. 206, 942-951 (2008)

9. Ohtsuka, H, Nakamura, S: On the sum of reciprocal Fibonacci numbers. Fibonacci Q. 46/47, 153-159 (2008/2009)

10. Holiday, S, Komatsu, T: On the sum of reciprocal generalized Fibonacci numbers. Integers 11, $441-455$ (2011)

11. Rabinowitz, S: Algorithmic summation of reciprocals of products of Fibonacci numbers. Fibonacci Q. 37, 122-127 (1999)

12. Ipek A: On the spectral norms of circulant matrices with classical Fibonacci and Lucas numbers entries. Appl. Math. Comput. 217, 6011-6012(2011) 
13. Bahsi, M, Solak, S: On the norms of $r$-circulant matrices with the hyper-Fibonacci and Lucas numbers. J. Math. Inequal. 8, 693-705 (2014)

14. Bozkurt, D: On the spectral norms of the matrices connected to integer number sequences. Appl. Math. Comput. 219, 6576-6579 (2013)

15. Kocer, EG, Mansour, T, Tuglu, N: Norms of circulant and semicirculant matrices with Horadam's numbers. Ars Comb. $85,353-359$ (2007)

Submit your manuscript to a SpringerOpen ${ }^{\circ}$ journal and benefit from:

- Convenient online submission

Rigorous peer review

- Immediate publication on acceptance

- Open access: articles freely available online

- High visibility within the field

- Retaining the copyright to your article

Submit your next manuscript at $\boldsymbol{s p r i n g e r o p e n . c o m ~}$ 